\title{
Towards an Integrated Model of Data Governance and Integration for the Implementation of Digital Transformation Processes in the Saudi Universities
}

\author{
Abdulfattah $\operatorname{Omar}^{1}(\mathbb{\square})$ \\ Department of English \\ College of Sciences \& Humanities \\ Prince Sattam Bin Abdulaziz University
}

\author{
Ahmed Almaghthawi ${ }^{2}$ \\ Department of Computer Science and Artificial Intelligence \\ University of Jeddah \\ Jeddah, Saudi Arabia
}

\begin{abstract}
In the face of the challenges of the Digital Age and the outbreak of the pandemic COVID-19 which have changed higher education institutions remarkably, universities are urgently required to speed up their digitalization initiatives and cope up with the global digital developments to survive. For the successful implementation of digital transformation, however, data governance should be considered. Despite the extensive literature on data governance and digital transformation, there is no focus on the issue in the Saudi Higher Education institutions. In the face of this, the current study investigates data governance policies and practices in the Saudi Universities. This study is built on a case study design. Nine universities in Saudi Arabia were selected for the purpose of the study. These include public, community, and private universities that make up the Higher Education system in Saudi Arabia. For data collection purposes, three methods were selected. These were the survey, focus group discussions and in-depth interviews. The findings of this study indicate that data governance is an effective tool in the implementation of digital transformation processes in higher education institutions and thus should be embedded into strategies of the universities for using digital technologies in appropriate manners. Good data governance practices are required for smooth and effective digital transformation. Universities are required to create an effective functional team for the data governance tasks, develop an internal audit of data governance, follow-up the regulatory compliance procedures, define the priorities of data governance activities, provide frequent data governance training for employees and faculty members, set enforcement and follow-up standards, and conduct frequent assessments of data governance plans and policies. Although the study is limited to the Saudi universities, results and implications of this study can be a useful reference to choose effective data governance practices that can be successfully adopted and implemented to effectively manage critical information and use it to transform their day-to-day operations.
\end{abstract}

Keywords-COVID-19; data governance; digital transformation; higher education; Saudi Arabia

\section{INTRODUCTION}

The recent years have witnessed unprecedentedly rapid changes that have their impact on the global economy today [1, 2]. In response to these changes, businesses, corporations,

Paper Submission Date July 27, 2020

Acceptance Notification Date: August, 13, 2020

${ }^{\triangle}$ Corresponding Author. institutions, and even governments have realized the importance of adopting a change culture that enables them to be more willing to implement required changes and initiatives effectively and efficiently so that they can survive and remain competitive within this fast-changing business environment [3]. In this regard, different digital transformation initiatives have been adopted by organizations and institutions in both public and private sectors in different countries around the world. It is almost agreed that digital transformation is imperative for optimizing processes and making workflows faster, easier, and more efficient [4-6].

The Kingdom of Saudi Arabia has recently adopted some initiatives in relation to the digital transformation of the government sectors including education, healthcare, and telecommunications. These initiatives seek to improve operations and business value with the ultimate goal of achieving sustainable development and global effectiveness, increase the contribution of the digital economy to GDP, and improve the overall quality of life. The Digital Transformation national plan was designed to be carried out over three stages. The first stage lasted from 2005 to 2010; the second from 2012 through 2016; the third, currently in effect, began in 2019 and to be completed roughly in 2022; its main components are digital health, digital education, e-commerce, and smart cities.

In education, great efforts have been recently made in order to achieve the goals of the digital transformation clearly stated in the strategic objectives of the Saudi Vision 2030. In this regard, the Ministry of Education launched the Future Gate project for implementing digital transformation processes in all the educational institutions in the Kingdom. The project seeks to convert paper curricula into digital, and work to convert traditional classes into smart ones. The project was launched in collaboration with the TETCO Company for educational technologies in 2018.

The project is considered by many as one of the most promising projects in the field of education, especially with the rapid growth of its beneficiaries. It aims to create a good and enjoyable learning environment in order to reduce the Kingdom's traditional environment for education. With digital education, a positive interaction can be created between students on the one hand, and students and instructors on the 
other by means of modifying the traditional style of education, and replacing it with digital education.

With the emergence of the Coronavirus crisis known as COVID-19, it was clear that the shift into digital transformation in education is imperative. Educators stressed the importance of developing a well-built infrastructure that can accommodate and facilitate the digital transformation processes in education. Taken the consequences of COVID-19 into consideration, the digital transformation of higher education has to be accelerated [7]. It is now important to accelerate digital transformation processes and these cannot be delayed in any way [8].

For the successful implementation of digital transformation processes, however, data governance policies and procedures need to be developed and enforced [9]. In spite of the importance of the issue, very little has been done on data governance in the Saudi institutions in general and higher education institutions and universities in particular. This study addresses this gap in the literature through proposing a model for data governance in the Higher Education institutions in Saudi Arabia. In order to achieve the study objectives, a questionnaire survey was designed and in-depth interviews and discussion focus-groups were conducted with the IT departments in 7 Saudi Higher Education institutions and universities. A mixed approach of quantitative and qualitative methods was adopted in order to evaluate the data governance policies and practices adopted by the selected institutions.

The remainder of this article is organized as follows. Section 2 is a brief survey on the emergence of digital transformation and the relationship between digital transformation on one side and data governance and integration on the other. Section 3 describes the methods and procedures. Section 4 is an analysis of the data. Section 5 summarizes the findings of the study and discusses their implications to higher education institutions.

\section{LITERATURE REVIEW}

Digital transformation refers simply to change processes whereby a shift is brought about so that technical and digital developments are replacing traditional and conventional methods for faster and better performance [10, 11]. Digital transformation provides huge potentials to build effective, competitive and sustainable societies, by achieving a fundamental change in the services of various parties, consumers, employees and beneficiaries, while improving their experiences and productivity through a series of commensurate processes, accompanied by a reformulation of the necessary procedures for activation and implementation [11, 12]. Digital transformation requires enabling a culture of creativity as well as a well-built infrastructure that accommodates any required changes in the work environment, from infrastructure, operating models, to marketing services and products $[9,13$, 14].

According to Fenton, Fletcher, and Griffiths, digital transformation has the potentials of reshaping the way people live, work, think, interact and communicate with people, depending on available technologies, with continuous planning and a constant quest to reformulate practical experiences [15].
In this way, successful digital transformation processes can improve efficiency, reduce spending, and implement new services quickly and flexibly. They have the effect of achieving a fundamental change in the services provided to individuals in different fields including health, education, safety and security, and improving their experiences and productivity [13].

The literature suggests that digital transformation has been key in almost all innovation and change processes adopted by institutions and organizations due to its positive impacts on increasing workflow efficiency and reducing error, improving performance productivity, and quality, and thus enhancing customer satisfaction $[14,16]$.

In order for successful implementation of digital transformation, however, some factors need to be considered $[17,18]$. These include data governance and integration. In fact, data is one of the most critical elements in all digitalization processes; therefore, it must be correct, complete, and accurate [19]. Practically every process within an organization relies on data to operate effectively, so the organization's strategy of data governance impacts the entire organization, not just the IT departments. Leignel, Ungaro, and Staar argue institutions should have effective, working and reliable data governance practices and policies in order to face the challenges of digital transformation. Through such practices and policies, organizations can effectively manage their investment in IT systems and make the most of their development [20].

Data governance can be defined as the concept of managing data and information to produce high-quality data, clean, accurate, and safe data [21, 22]. It is an umbrella term that encompasses processes of the creation, storage, use, evaluation, archiving and deletion of information [23, 24]. It includes processes, roles, policies and standards that ensure the effective and efficient use of information, and in that way it enables institutions to achieve their goals effectively and efficiently [21].

Data governance is based on many processes that are associated with the life cycle of information and data. These include data creation, data organization, data storage, data backup and recovery, data management and maintenance, and finally data retention and secure destruction. Organizations need to have a comprehensive and integrated data governance strategy that helps them maximize the value of their digital information assets while minimizing potential risks. In this sense, data governance is designed to guide records management and corporate content management, not replace it, while also provide a framework for making smarter decisions regarding valuable information and where it is best stored within the IT infrastructure [19].

Based on the critical role of data in digitalization processes, it is almost agreed that data governance is indispensable to digital transformation processes for all organizations including universities today [20]. Given the tremendous growth in the amount of data that universities today create, collect and store, the concept of data governance has increased in recent years. Universities have realized the need to establish laws governing this vast amount of data and information in addition to their 
fear from the risk of security, privacy, compliance and legal violations.

Universities are also encouraged to adopt working data governance policies in order to make use of what has come to be known as Big Data - which can represent great opportunities for universities and educational institutions if they are properly employed. Through effective data governance practices, universities and educational institutions can move faster, make smarter decisions, and provide better insights into consumer behavior, which will contribute to increased efficiency. Before the emergence of what was known as big data, decisions regarding how to store data were mainly made by the employees and individuals who created and used the data. Many universities have realized that they need a more structured structure and central control that oversees how data is stored, managed, kept and protected from violation whether unintentionally or by penetration of sensitive information.

With the increasing importance of data in almost all university operations today, data governance remains a major issue. Nevertheless, universities still have difficulties in terms of data management which definitely has negative impacts on its processes. These difficulties are generally attributed to poor data governance. In light of this argument, this study seeks to propose an integrated model of data governance for successful data management and proper implementation of digital transformation processes in the Saudi Universities.

\section{METHODS}

This study is built on a case study design. Nine universities in Saudi Arabia were selected for the purpose of the study. These include public, community, and private universities that make up the Higher Education system in Saudi Arabia. The selected institutions represent the three categories. For data collection purposes, three methods were selected. These were the survey, focus group discussions and in-depth interviews. For data analysis, a sequential mixed method design using a combination of quantitative and qualitative approaches.

The employees, faculty members, executives and stakeholders in this study represent the population. The sampling was done in the form of survey participants and interviewees, which was an appropriate selection from the whole population. It is always difficult to include the whole population, hence a typical sample was only chosen for the purpose of generalizing the result to the population. For data representativeness reasons, the survey was publicized through all possible means in order to reach a maximum number of employees and stakeholders using the universities' services so that the findings and results should be interpreted and generalized as representing the views of employees and stakeholders. 477 responded to the survey. These represent different employment levels and stakeholders. In the qualitative phase of this study, six senior members (two of each institution), holding the positions of manager and above levels, were interviewed and 27 other employees were sampled for the informal focus groups as shown in Table I. All the participants in the interviews and discussion groups had also responded to the survey so it was easy to triangulate the findings of the survey with the observations during the interviews and focus groups. The data collected through a survey questionnaire was triangulated through transcripts of the Focus groups, interviews, as well as institutional documentation and website material including its annual reports and shareholders' feedback. The data findings were underpinned with the established theories to determine whether the Saudi universities have the potentials to make the shift towards digital transformation smoothly and effectively. Interview and Focus Group Questions were both close-ended and open-ended in which very short text answers were expected.

TABLE I. Detalls about THE Data Sources

\begin{tabular}{|l|l|l|l|}
\hline Institutions & Survey & Focus Group & Interviews \\
\hline General & & - & - \\
\hline Top management & 60 & 3 & 3 \\
\hline IT staff & 105 & 9 & 3 \\
\hline Employees \& Faculty & 240 & 9 & - \\
\hline Other stakeholders & 72 & 6 & - \\
\hline Total & 477 & 27 & 6 \\
\hline
\end{tabular}

The questionnaire is in three main parts. In Part One, respondents were asked about their perceptions about data governance practices in their institution, how employees and stakeholders can be educated about data governance policies, and how the institutions can ensure the efficiency of the digital transformation processes in their institutions. In Section Two, the participants were also asked about data governance principles followed in their institutions, their frequency and employees' opinions about those principles. In Section Three, participants were asked whether good data governance practices had been in place to facilitate digital transformation processes.

The questions prepared for the interviews and focus groups were only guiding questions (about the subject of the study), but during the interview and discussion, a few probe questions were also added and supplemented to the main questions. This helped the researchers to cope with the respondents' stories and to get thick and detailed descriptions. All the participants had an opportunity to talk about their experiences in relation to data governance principles. Their narratives indicated their understanding of the data governance and its benefits which the researchers used in identifying the themes and subthemes for its content analysis.

\section{ANALYSIS AND Discussions}

The data collected from the questionnaire revealed interesting information about the application and implementation of data governance in the Saudi universities. The employees, faculty members, executives and stakeholders showed great familiarity with the principles of data governance. The data also revealed that data governance was deeply embedded in the universities' portfolios. This information was triangulated through the data collected from interviews and focus groups. The participants revealed almost a similar account of data governance being followed at their institutions and how these practices contributed to making the shift towards digital transformation smoothly and effectively. The researchers inferred from both the questionnaire of data 
and the narratives of participants that they had familiarized themselves with both data governance principles and also the manner they were being implemented and monitored. The interview respondents admitted that during the learning and training programs, they involved in self-reflection and collaborative discussions with one another for a better understanding of the data governance principles. Respondents also realize the importance of data governance practices and policies for a smooth and effective shift towards digitalization. Nevertheless, it is also clear that universities still have difficulties in terms of dealing with big data and making use of these data in ways that have positive impacts on the performance and quality of the organizations. Some executives reported that only $11 \%$ of their data is appropriately used. This means that approximately 90 percent of the digital information is stored as "dark data", unorganized, undisclosed and untapped.

The findings of the study agree with the data governance literature in relation to the effective role data governance can play in the implementation of digital transformation processes. Successful organizations have to adopt working data governance policies that ensure the effective creation of data and dealing with these data in an appropriate manner [18, 25]. The claim here is that organizations which do not have a governance strategy face critical risks while those which work within a governance framework perform measurably better [26]. It is also true that data governance and management are closely related to the performance of institutions and organizations. Big data should be considered as opportunities for growth, performance and competitiveness [27, 28]. Ramingwong and Manopiniwes stress that data governance and data security management are critical success factors for the productivity and survival of industries and organizations today [29].

In light of these findings, it is suggested that universities need to develop a virtual culture to meet the challenges of the digital age we witness today. This, however, requires both imaginative and creative implementation, as well as open and innovative leadership. Universities should devise strategic plans for digital transformation and that includes the selection of appropriate technologies taking into account training and experience. The components of these strategic plans are shown in Fig. 1.

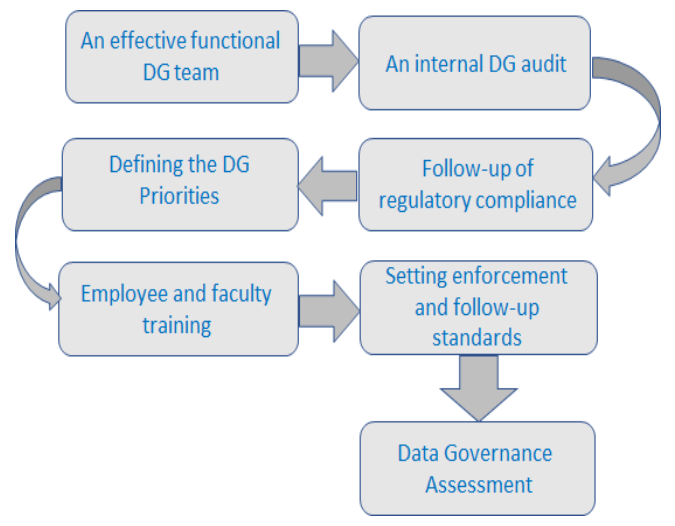

Fig. 1. A Proposed Model for Good Data Governance Practices in the Saudi Universities.
In the first place, each university should create an effective functional team for data governance tasks. No data governance plan will succeed if it does not reflect the needs and goals of all major stakeholders, including legal entities, compliance officers, risk management, human resources, information technology, and others [21,30]. Each of these entities should be represented at the initial planning stage and have a say in determining success factors, key outcomes and potential risks.

Second, universities should have an internal audit for data governance that can be usefully used for developing a robust information management framework [23, 31, 32]. This should have the effect of defining and identifying the main data sources universities interact with including outdated systems, and data archives that are potentially not actively managed at all.

Universities also need to carefully evaluate and follow-up the legal and regulatory requirements of data governance practices. It is extremely important to understand all external retention requirements, decide the duration data should remain and how long it will be kept and review these requirements often to ensure that the governance plan will be kept up to date [31]. Equally important, universities should define the priorities of data governance activities [33, 34]. Information governance plans are not created to be implemented overnight, so it is important to address the most urgent issues first. Data evaluation should define the priorities of primary information governance activities. For example, if a review reveals a large number of decentralized data stored randomly across a set of shared drives, a good first step might be to create a data map that connects employees and faculty members to the data sources they interact with frequently. Likewise, if the initial evaluation reveals the accumulation of a large number of unnecessary backup files, the data governance plan should focus more on developing and implementing a better deletion policy.

It is important for universities also to provide training programs for employees and faculty members on the best data governance practices since the successful implementation of digitalization processes relies heavily on the workforce. Training should not be confined to IT departments and personnel only [35, 36]. Employees and faculty members should understand how data governance policies and procedures affect their daily activities. It is also important to explain for them the importance of data and data governance policies and their role in the digital transformation processes [36-39]. Training programs should also be supplemented with enforcement and follow-up standards that ensure the implementation of data governance programs [21, 40]. Finally, universities need to conduct periodic and frequent assessments of data governance plans, activities, and policies. Assessments should consider the way the effectiveness of data governance programs can be proved and whether the data governance goals and objectives are achieved $[22,30]$.

\section{CONCLUSION}

The outbreak of the pandemic COVID-19 has accelerated the digitalization developments in many fields including higher education institutions. Universities now are urgently required to speed up their digitalization initiatives and cope up with the 
global digital developments in order to survive. For the successful implementation of digital transformation, however, data governance should be considered. This study addressed the nexus between data governance and digital transformation in higher education institutions. The findings of this study indicate that data governance is an effective tool in the implementation of digital transformation processes in higher education institutions and thus should be embedded into strategies of the universities for using digital technologies in appropriate manners. Good data governance practices are required for smooth and effective digital transformation.

Universities are required to create an effective functional team for the data governance tasks, develop an internal audit of data governance, follow-up the regulatory compliance procedures, define the priorities of data governance activities, provide frequent data governance training for employees and faculty members, set enforcement and follow-up standards, and conduct frequent assessments of data governance plans and policies. Although the study is limited to the Saudi universities, results and implications of this study can be a useful reference to choose effective data governance practices that can be successfully adopted and implemented to effectively manage critical information and use it to transform their day-to-day operations. Further research is required to provide higher education institutions with workable and reliable solutions for the proper management of big data and the assurance of data quality for improving operational efficiency.

\section{ACKNOWLEDGMENTS}

This publication was supported by the Deanship of Scientific Research at Prince Sattam bin Abdulaziz University, Alkharj, Saudi Arabia.

\section{REFERENCES}

[1] S. Furusten, Institutional Theory and Organizational Change. Edward Elgar Publishing, Incorporated, 2013.

[2] P. Myers, S. Hulks, and L. Wiggins, Organizational Change: Perspectives on Theory and Practice. OUP Oxford, 2012.

[3] D. C. Dunphy, A. Griffiths, and S. Benn, Organizational Change for Corporate Sustainability: A Guide for Leaders and Change Agents of the Future. Routledge, 2003.

[4] G. Westerman and D. Bonnet, Leading Digital: Turning Technology into Business Transformation. Harvard Business Review Press, 2014.

[5] N. Palmer et al., Digital Transformation with Business Process Management. Future Strategies Inc., 2019.

[6] S. Shariffuddin and J. Razali, "Transformation of university colleges to full-pledged universities: A proposed conceptual framework for Malaysian higher learning institutions," International Journal of Advanced and Applied Sciences, vol. 4, no. 12, pp. 168-173, 2017.

[7] S. Martin-Barbero, "COVID-19 has accelerated the digital transformation of higher education," World Economic Forum21 Jul 20202020 , Available: https://www.weforum.org/agenda/2020/07/covid-19-digitaltransformation-higher-education/.

[8] M. D. P. Uys, One World One School: Education's Forthcoming Fundamental Transformation. Independently Published, 2020.

[9] A. Maheshwari, Digital Transformation: Building Intelligent Enterprises. Wiley, 2019.

[10] T. U. Daim, Digital Transformation: Evaluating Emerging Technologies. World Scientific Publishing Company Pte Limited, 2020.

[11] B. Daniotti, M. Gianinetto, and S. D. Torre, Digital Transformation of the Design, Construction and Management Processes of the Built Environment. Springer International Publishing, 2019.
[12] A. Gilchrist, Digital Success: A Holistic Approach to Digital Transformation for Enterprises and Manufacturers. alasdair gilchrist, 2018.

[13] V. Kale, Digital Transformation of Enterprise Architecture. CRC Press, 2019.

[14] D. R. A. Schallmo and C. A. Williams, Digital Transformation Now!: Guiding the Successful Digitalization of Your Business Model. Springer International Publishing, 2018.

[15] A. Fenton, G. Fletcher, and M. Griffiths, Strategic Digital Transformation: A Results-Driven Approach. Taylor \& Francis, 2019.

[16] T. Saldanha, Why Digital Transformations Fail: The Surprising Disciplines of How to Take Off and Stay Ahead. Berrett-Koehler Publishers, 2019.

[17] B. Borden, C. Fudge, J. Nelson, J. Porell, and I. Redbooks, Accelerating Digital Transformation on Z Using Data Virtualization. IBM Redbooks, 2018.

[18] P. Jackson and C. Carruthers, Data Driven Business Transformation: How to Disrupt, Innovate and Stay Ahead of the Competition. Wiley, 2019.

[19] J. M. Barker, Data Governance: The Missing Approach to Improving Data Quality. University of Phoenix, 2016.

[20] J. L. Leignel, T. Ungaro, and A. Staar, Digital Transformation: Information System Governance. Wiley, 2016.

[21] J. Ladley, Data Governance: How to Design, Deploy and Sustain an Effective Data Governance Program. Elsevier Science, 2012.

[22] U. Gupta and S. Cannon, A Practitioner's Guide to Data Governance: A Case-Based Approach. Emerald Publishing Limited, 2020.

[23] H. Sen, Data Governance: Perspectives and Practices. Technics Publications, 2019.

[24] B. van Gils, Data Management: a gentle introduction: Balancing theory and practice. Van Haren Publishing, 2020.

[25] F. P. G. Márquez and B. Lev, Big Data Management. Springer International Publishing, 2016.

[26] Y. Y. Haimes and A. P. Sage, Risk Modeling, Assessment, and Management. Wiley, 2015.

[27] Y. Yuan and C. Ho, "Needs analysis of national parks for applying big data solutions in tourism management," International Journal of Advanced and Applied Sciences, vol. 4, no. 12, pp. 20-36, 2017.

[28] W. Lee, A. Chong, and T. Ramayah, "Organizational culture and performance of Malaysian manufacturing firms," International Journal of Advanced and Applied Sciences, vol. 5, no. 12, pp. 59-66, 2018.

[29] S. Ramingwong and W. Manopiniwes, "Supportmentfor organization and management competences of ASEAN community and European Union toward Industry 4.0," International Journal of Advanced and Applied Sciences, vol. 6, no. 3, pp. 96-101, 2019.

[30] D. Cervo, M. Allen, and J. Dyché, Master Data Management in Practice: Achieving True Customer MDM. Wiley, 2011.

[31] R. D. McDowall, Data Integrity and Data Governance: Practical Implementation in Regulated Laboratories. Royal Society of Chemistry, 2018.

[32] H. Elbardan and A. O. Kholeif, Enterprise Resource Planning, Corporate Governance and Internal Auditing: An Institutional Perspective. Springer International Publishing, 2017.

[33] D. B. A. M. Khosrow-Pour, Encyclopedia of Information Science and Technology, Fourth Edition. IGI Global, 2017.

[34] D. B. A. M. Khosrow-Pour, Advanced Methodologies and Technologies in Business Operations and Management. IGI Global, 2018.

[35] N. Bhansali, Data Governance: Creating Value from Information Assets. CRC Press, 2013.

[36] E. M. Power and R. L. Trope, Sailing in Dangerous Waters: A Director's Guide to Data Governance. American Bar Association, Section of Business Law, 2005.

[37] S. K. Strydom and M. Strydom, Big Data Governance and Perspectives in Knowledge Management. IGI Global, 2018.

[38] R. Mahanti, Data Quality: Dimensions, Measurement, Strategy, Management, and Governance. ASQ Quality Press, 2019. 
[39] A. D. Giordano, Performing Information Governance: A Step-by-step Guide to Making Information Governance Work. Pearson Education, 2014.
[40] R. S. Seiner, Non-Invasive Data Governance: The Path of Least Resistance and Greatest Success. Technics Publications, 2014. 